Arch. Tierz., Dummerstorf 42 (1999) 5, $481-493$

Aus dem Forschungsinstitut fur die Biologie landwirtschaflicher Nutztiere (FBN), Dummerstorf ' und dem
Forschungszentrum für Biosystemtechnik und Biomaterialien Rostock - WarnemÜnde ${ }^{2}$

HARTMUT FRANZ ${ }^{1}$ und HORST REICHART ${ }^{2}$

\title{
Der Feldermonitor - eine neue Möglichkeit der Lernforschung mit Tieren und Ergebnisse bei visuellen Differenzierungsaufgaben von Zwergziegen
}

\section{Summary}

Title of the paper: A general purpose computer system for behavioral conditioning experiments, named 'Feldermonitor' - a new possibility for learning experiments with animals and results of visual discrimination tasks in dwarf goats

The precise and automatised testing of sensoric as well as cognitive abilities of pets and livestock becomes increasingly important regarding both, the objecitve evaluation of their welfare and the develpoment of new automatic animal management facilities. The learning apparatus, named Feldermonitor and introduced here, proved to be a valuable apparatus for learning experiments allowing continuous testing of learning behaviour of animals under group as well as individual housing conditions. The developed hard and soft ware enables the tested animals to choose simultaneously among up to 12 options, which will confront them with a maximum of four elements of rewarding, informing or sanctioning.

The experiments described were performed with dwarf goats. The animals were tested using simultaneous discrimination tasks with two or four stimuli and being rewarded with small portions of water after each success. Comparing mean learning curves it can be shown, that in case of consecutive learning tests, successful learning starts earlier as well as faster with each additionally performed test. The rapidity of learning increases also, if different discrimination tasks are offered consecutively. The coefficient of variation for learning success among animals was 10 to $20 \%$, indicating high individual differences.

Key words: learning, apparatus, computer, visual discrimination, dwarf goat, group-housing

\section{Zusammenfassung}

Eine exakte und weitgehend automatisierte Prüfung von sensorischen oder kognitiven Fähigkeiten von Heimund Nutztieren erhält sowohl im Hinblick auf eine objektive Beurteilung ihrer Befindlichkeit als auch für die Entwicklung neuer, automatisierter Tierversorgungseinrichtungen eine zunehmende Bedeutung. Die diesbezüglich entwickelte Lernapparatur „Feldermonitor" erwies sich für kontinuierliche Prüfungen des Lernverhaltens von Tieren bei Gruppen- oder Einzelhaltung als geeignet. Die damit verfuigbare Hard- und Software gestattet die simultane Nutzung von maximal 12 Wahlschaltern durch die Tiere und von maximal 4 verschiedenen Belohnungs-, Informations- oder Bestrafungseinrichtungen.

In den vorgestellten Experimenten wurden Zwergziegen in verschiedenen, visuellen Differenzierungsaufgaben bei zweifach oder vierfach Simultanwahl und einer Belohnung durch Wasserportionen geprüft. Der Vergleich der mittleren Lernkurven zeigt, daß bei den aufeinander folgenden Lemtests der Anstieg des Lernerfolges von Test zu Test früher einsetzt und steiler wird. Die Lerngeschwindigkeit wird bei aufeinander folgenden, verschiedenen Differenzierungsaufgaben größer. Zwischen den Tieren bestanden beträchtliche Unterschiede im Lernerfolg. Der Variationskoeffizient dafür lag zwischen 10 und $20 \%$.

Schlüsselwörter: Lernen, Apparatur, Computer, visuelle Differenzienung, Ziege, Gruppenhaltung 


\section{Einleitung}

Eng verbunden mit der beachtlichen Entwicklung der Computertechnik im letzten Viertel dieses Jahrhunderts und den Fortschritten in der ethologischen und neurobiologischen Forschung sind die Bemühungen, auch in der Lernforschung mit Tieren moderne Technologien nutzbar zu machen. Der Einsatz von Computern und Videogeräten in Lernexperimenten begann Ende der 80-er Jahre zunächst in der Primatenforschung. DASSER (1987), WASHBURN und RUMBAUGH (1991), WASHBURN et al. (1994) und HOPKINS et al. (1996) nutzten Computer und Video- bzw. Diaprojektionen, um das Wahlverhalten von Affen (Macaca fascicularis, Macaca mulatta, Pan troglodytes) zu untersuchen. Alle Autoren kamen zu dem Ergebnis, daß die genutzten Lernapparaturen für Einzelprüfungen gut geeignet seien.

FEULNER (1993) untersuchte mit einer computergestützten Lernapparatur an einem Mäusestamm (Han:NMRI) den Einfluß von Transferlernen auf die Stabilität der Verknüpfung sensorischer und motorischer Gedächtnisinhalte. Die Mäuse hatten jeweils die Wahl zwischen zwei Mustern und vier Bewegungsrichtungen eines Schalthebels, der dem Muster zugeordnet war. Alle Aktionen wurden vom Computer gespeichert. Bei Richtigwahl erfolgte durch den Computer die Ansteuerung des Futterautomaten. Der Autor kam zu dem Schluß, daß die Tiere, die einen sensorischen Transfer absolvierten, auch ihr in der Konditionierungsphase gelerntes motorisches Bewegungsmuster änderten, obwohl die motorische Aufgabe beim Transfer dieselbe blieb. Feulner nutzte den Computer als Registrier- und Steuereinheit und testete die Tiere einzeln.

COOK et al. (1995) und EMMERTON (1998) prüften Tauben an einem Computer auf ihre Fähigkeit, Gleich-Ungleich-Differenzierungsaufgaben und Farbdifferenzierungsaufgaben mit unterschiedlicher Darbietungsform zu lösen. Auf Farbmonitoren wurden unterschiedliche Stimuli erzeugt und die Schnabelhiebe der Tauben mittels Infrarot LED Touchscreen gezählt. Die Autoren kamen zu dem Schluß, daß die gewählten Apparaturen für die Einzelprüfung von Tauben geeignet sind.

ANDREWS und JANSEN (1996) und ENNACEUR et al. (1997) untersuchten Ratten im Einzeltest (Long Evans, PVG strain) an computergestützten Wahlanlagen. Die Ratten hatten bei ANDREWS und JANSEN die Möglichkeit, durch Annäherung (nosepokes) an zwei durch Photozellen kontrollierte und durch Projektoren erzeugte visuelle Stimuli eine Futterbelohnung zu erhalten, wenn sie den richtigen Stimulus wählten. Bei Falschwahl folgten 15 Sekunden Finsternis. Von den 16 Tieren erreichten 6 nicht das Trainingsziel, weil sie entweder die Stimuli nicht berührten oder aber eine Seitenstetigkeit entwickelten und dem S+ Stimulus nicht folgten. ENNACEUR et al. kamen zu dem Schluß, daß ihre Anlage durch Screen Monitore weiter zu verbessern wäre und sich für weitere Untersuchungen des Erinnerungsvermögens eigne.

MEIER et al. (1998) entwickelten eine computergestützte, automatische Lernapparatur für in Gruppen gehaltene Gerbils (Meriones unguiculatus). Die Apparatur gestattet jeweils einem Tier die Auswahl einer von zwei, mit unterschiedlichen visuellen Stimuli gekennzeichneten Türen, um an einen Futterautomaten zu kommen. Die Autoren nennen als Hauptvorteile der Apparatur die Vermeidung von sozialer Deprivation und die Möglichkeit der selbständigen Entscheidung der Tiere über Zeitpunkt und Häufig- 
keit der Besuche.

Mit diesen Arbeiten wurde die Möglichkeit der Nutzung des Computer Monitors, verschiedener Displays und von Bild- und Videoprojektionen in der Lernforschung mit Labor- und Zootieren begründet.

Mit Ausnahme der von WASHBURN et al. (1994) und von MEIER et al. (1998) vorgestellten Lernapparaturen eignen sie sich nicht für die Gruppenprüfung von Tieren. Die meisten der vorgestellten Apparaturen setzen eine zeitlich begrenzte Einzelhaltung des zu prüfenden Tieres an der Lernapparatur voraus.

Daraus können sich Nachteile ergeben, wie Isolationsstreß für das Tier, störende Tageszeiteffekte, eingeschränkte Möglichkeiten zur Untersuchung von Wechselwirkungen des Lern- und Sozialverhaltens sowie ein höherer Aufwand (Personal, Zeit, Kosten).

Um in Gruppen gehaltene Tiere über längere Zeiträume prüfen zu können, erscheint die Schaffung folgender wichtiger Voraussetzungen erforderlich.

Beispielsweise sollte die eigentliche Lernapparatur so in die Haltungsumwelt integriert werden können, daß sie mittels frei zugänglicher Einzeltierstände von allen Tieren beliebig oft und leicht erreicht werden kann. Eine Beeinflussung des Prüflings durch andere Tiere ist dabei auszuschließen. Durch den Einsatz von automatischen Tiererkennungssystemen sollte die kontinuierliche Erfassung und Auswertung von Einzeltierdaten gesichert werden. Die eingesetzten Tierversorgungseinrichtungen dürfen den $\mathrm{Be}-$ trieb der Lernapparatur nicht stören. Eine diesen Zielen entsprechende Lernapparatur sollte sich durch Anpassung der peripheren Einrichtungen für verschiedene Tierarten und für unterschiedliche Ziele nutzen lassen.

Das Ziel unserer Arbeit bestand in der Entwicklung einer neuen, computergestützten Lernapparatur, die sich für eine teilautomatisierte Prüfung von Tieren in Gruppenhaltung eignet. Für diese Apparatur war zu prüfen, ob Zeitpunkt, Art und Häufigkeit von Wahlversuchen durch die Tiere einen störungsfreien Ablauf von Lerntests für alle beteiligten Tiere der Gruppe gewährleisten. Es sollten das individuelle Wahlverhalten, Mittelwert und Standardabweichung für Parameter des Lernverhaltens im Testverlauf bei verschiedenen visuellen Differenzierungsaufgaben geprüft und auf dieser Basis die Eignung der Lernapparatur Feldermonitor für die experimentelle Lernforschung beurteilt werden.

\section{Methodik und Tiere}

In einer $12 \mathrm{~m}^{2}$ großen Haltungsbox wurden zunächst 11 weibliche und später 10 männliche Jungtiere der Versuchstierpopulation afrikanischer Zwergziegen (Capra hircus L.) des FBN Dummerstorf gehalten. In der Box wurde ein Einzeltierstand für die Lernapparatur aufgestellt. Er ist in den Abbildungen 1 und 2 skizziert bzw. abgebildet.

Die Maße des Tierstandes waren so gewählt, daß nur ein Tier den Stand betreten und sich ungestört darin aufhalten konnte. An der Stirnwand des Standes befand sich der Computermonitor hinter einer durchsichtigen Scheibe. Eine der beiden Seitenwände des Standes bestand aus durchsichtigem Material, um den Tieren im Stand den Sicht- 


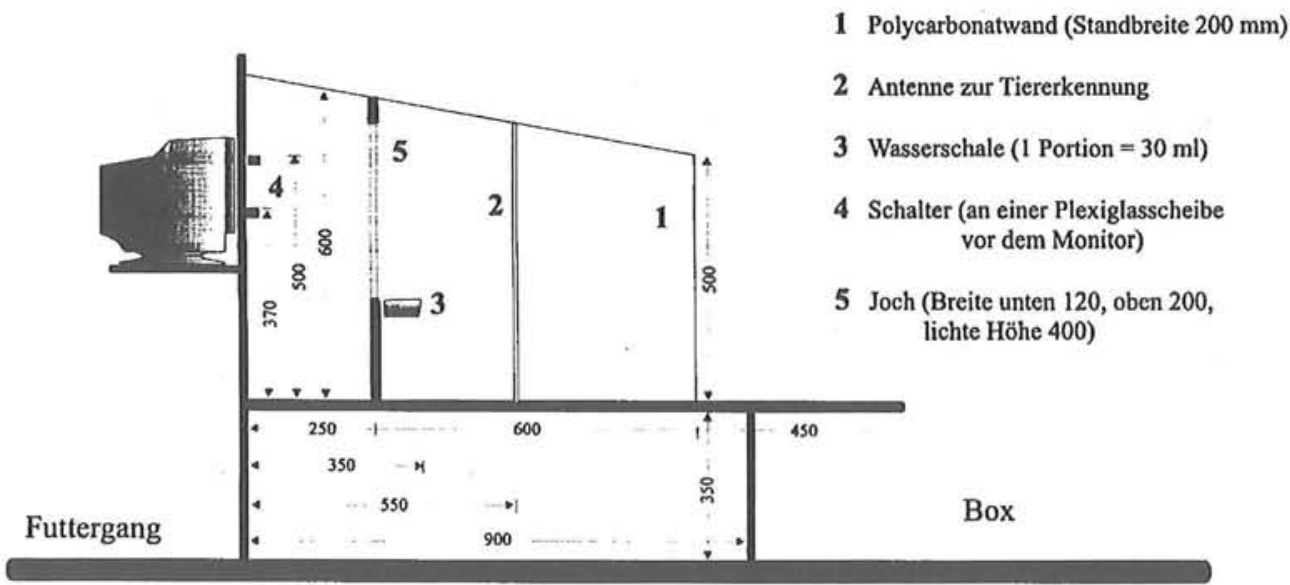

Abb. 1: Maße des Tierstandes für den Feldermonitor (Measurements of the box for the field monitor)

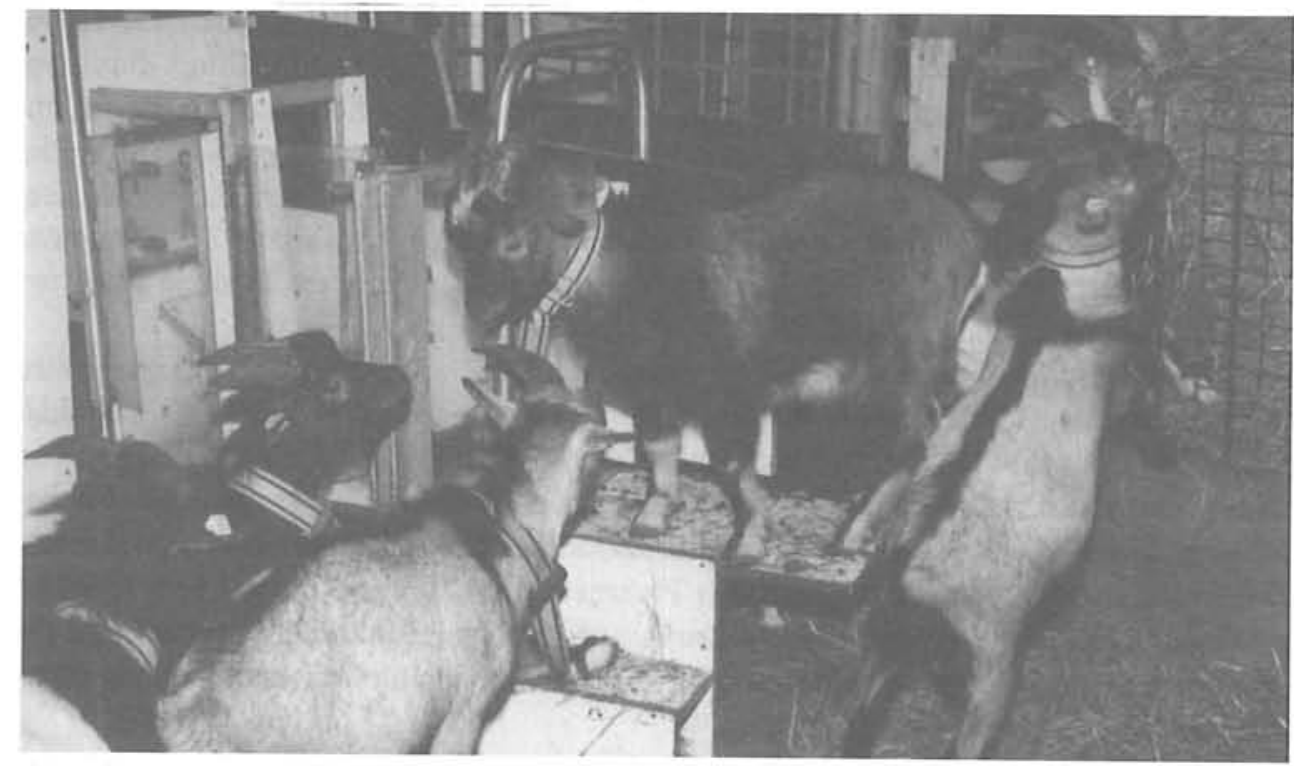

Abb. 2: Jungziegen am Feldermonitor (Versuchsgruppe 2, Test 3) (Young goats at the field monitor (experimental group 2 , test 3))

kontakt zur Gruppe zu ermöglichen. Außerdem wurden dadurch eine natürliche Belichtung und eine ständige Beobachtungsmöglichkeit gesichert. Die zweite Seitenwand (beschichtete Spanplatte) war verstellbar, so daß die Standbreite von Zeit zu Zeit der Größe der wachsenden Tiere angepaßt werden konnte. Von oben war der Stand durch ein Brett abgedeckt, damit nachdrängende Tiere nicht aufspringen konnten. Auf dem Fußboden des Standes war ein Brett in Längsrichtung so angebracht, daß die Tiere dieses Brett zwischen den Beinen hatten. Dadurch war ein Unterlaufen des im Stand befindlichen Tieres von hinten ausgeschlossen.

Die Tiere trugen Halsbänder mit Respondern für die individuelle Erkennung mittels 
Antenne (s. Abb. 1, Punkt 2). Um die an einer Scheibe vor dem Monitor angebrachten Schalter (Punkt 4) berühren zu können, mußten die Tiere ihren Kopf durch ein Joch stecken (Punkt 5). Damit wurde ein Umdrehen der Tiere im Stand ausgeschlossen. Bei Richtigwahl erhielten die Tiere computergesteuert eine Portion Wasser $(30 \mathrm{ml})$ in eine vor dem Joch angebrachte Wasserschale. Die Schale war etwa $35 \mathrm{~cm}$ vom Bildschirm entfernt. Dadurch wurden die Tiere veranlaßt, nach der Richtigwahl etwas zurückzutreten, das Wasser zu trinken und dann wieder auf den Bildschirm zuzugehen. Damit wechselte der Augenabstand zum Bildschirm und das Tier konnte die für die Erkennung beste Entfernung wählen. Die Höhe der oberen Schalter sollte der Augenhöhe bei normaler Kopfhaltung entsprechen.

$\mathrm{Zu}$ jedem Schalter war auf dem dahinterliegenden „Feld“ des Monitors ein Symbol abgebildet. Im Lerntest 1 standen zwei und in den Lerntests 2 und 3 je vier Felder mit unterschiedlichen Symbolen zur Auswahl. Die verwendeten schwarz/weiß Symbole sind in der Tabelle 1 dargestellt. Ihre Position auf dem Bildschirm wurde nach jeder Schalterbetätigung gewechselt. Im Test 1 nach der Folge links, rechts, links, rechts usw., in den Tests 2 und 3 nach einer eingeschränkten Pseudozufallszahlenreihe. Dementsprechend wechselte auch die Steuerungsfunktion der zugehörigen Schalter. Nach jeder Schalterbenutzung wurde der Bildschirm für 3 Sekunden schwarz. Während dieser Pausenzeiten erfolgte Schalterbenutzungen führten zu keiner Reaktion des Apparates, wurden aber registriert und ausgewertet.

Tabelle 1

Zufalls-Erfolgswahrscheinlichkeit $\left(\mathrm{p}_{\mathrm{o}}\right)$ und visuelle Stimuli, die in den Lerntests eingesetzt wurden (Random probability for correct choice $\left(\mathrm{p}_{\mathrm{o}}\right)$ and visual stimuli used in the learning tests)

\begin{tabular}{ccccc}
\hline Test Nr. & $\mathrm{p}_{\mathrm{o}}$ & $\mathrm{S}+$ & $\mathrm{S}-$ & Bemerkung \\
\hline 1 & 0,5 & + & $\mathrm{O}$ & akustische Verstär- \\
& & & & kung \\
2 & 0,25 & + & T,,$\nabla$ & dto \\
3 & 0,25 & $\mathrm{~S}$ & H, A, N & dto \\
\hline
\end{tabular}

Die Symbole wurden mit dem Programm Paintbrush erstellt und als Bitmap Dateien in der Software für den Feldermonitor eingesetzt. Die Höhe und Breite der Symbole war $55 \mathrm{~mm}$, die Strichbreite $12 \mathrm{~mm}$. Zur akustischen Verstärkung jeder Schalterbetätigung wurden vom Steuerprogramm Wave Dateien genutzt. Für die Verstärkung der Wahl des S+ Stimulus („Richtigwahl“, Belohnung mit einer Wasserportion) erklang der Kammerton A. Bei einer Wahl des S- Stimulus („Falschwahl") erklang eine Dissonanz der Töne H, C, D. Die Töne erklangen unmittelbar bei Schalterbetätigung.

Die Software zur Steuerung der Lernapparatur und zur Erfassung der Daten für die Auswertung basiert auf dem Betriebssystem DOS 5.0 oder höher, WINDOWS 3.11 oder 95 und ist in VISUAL BASIC 3.0 modular aufgebaut. Als Computer wurde für den Einzeltierstand ein PC AT 486 DX-33 mit 4 MB RAM und 20 MB freiem Festplattenspeicher, SVGA Grafikkarte und einer Zusatzkarte zur zeit- und zustandsabhängigen Ausgabe von Steuerungssignalen eingesetzt. Mit dieser Hard- und Software ist das Erkennen der Betätigung von 1 - 12 Schaltern und das daran gebundene Ansteuern von 1 - 4 unterschiedlichen, peripheren Geräten zur Belohnung, Information oder Bestrafung möglich. 
In den zu beschreibenden Lerntests wurde die Belohnung durch Wasserportionen erreicht, für deren Regulierung ein Magnetventil vom Computer angesteuert wurde.

Für die von den Tieren zu benutzenden Schalter vor dem Monitor wurden Pegelschalter mit Reedkontakten genutzt. Die elektronische Tiererkennung erfolgte mit dem handelsüblichen System Megastar der Firma Urban, Wüsting, das durch Schnittstellenwandler ergänzt worden ist.

Der Einzeltierstand war mit Ausnahme geringer Wartungszeiten 24 Stunden am Tag geöffnet und von $6^{\circ \circ}-22^{\circ \circ} \mathrm{Uhr}$ beleuchtet.

Die Versuchstiere waren Zwergziegen aus der Zucht des Forschungsinstituts Dummerstorf (FBN). Die Elternpopulation wurde durch systematische Fremdverpaarung Afrikanischer Zwergziegen aus 7 deutschen Tiergärten erhalten. Diese heterogene Auszuchtpopulation erscheint für die Erzeugung repräsentativer Versuchstiergruppen geeignet. In die im folgenden beschriebenen Lernexperimente wurden 21 Lämmer aus der Herbstlammperiode 1997 einbezogen. Eine Übersicht zu den Versuchsgruppen gibt Tabelle 2.

Tabelle 2

Zwergziegen, die in die Lerntests einbezogen wurden (Dwarf goats used in the learning tests)

\begin{tabular}{cccccc}
\hline Versuchsgruppe & Geschlecht & Anzahl & $\begin{array}{c}\text { Geburtszeit- } \\
\text { raum }\end{array}$ & $\begin{array}{c}\text { kg-Geburts- } \\
\text { masse }\end{array}$ & $\begin{array}{c}\text { kg-Körper- } \\
\text { masse, vollend. } \\
\text { 6. Leb.-Mon. }\end{array}$ \\
\hline 1 & weiblich & 11 & $16 .-24.10 .97$ & 1,9 & 17,6 \\
2 & männlich & 10 & $21 .-29.10 .97$ & 2,3 & 18,9 \\
\hline
\end{tabular}

Die Lämmer wurden in den ersten vier Wochen nach der Geburt von ihren Müttern gesäugt und hatten den gesamten Stallbereich und oft auch die Fläche vor dem Stall als Auslauf. In der 5. Lebenswoche wurden sie an einen Tränkautomaten der Firma Urban, Wüsting abgesetzt und von diesem mit Milch semi ad libitum versorgt. Die Körpermassezunahme lag im Durchschnitt bei 80 - $90 \mathrm{~g}$ je Tag. Die Entwicklung der Körpermasse war auch während der Lerntests sehr gleichmäßig und erscheint normgerecht (Abb. 3). Zum Vergleich sind die Ergebnisse von PETZOLD (1980) und JACOB (1995) für den 180. Lebenstag eingefügt.

Die Milchversorgung mit dem Tränkautomaten erfolgte durch Dosierung von Tränkeportionen a $30 \mathrm{ml}$, die nach Betätigung eines Druckschalters in ein Tränkschälchen abgegeben wurden. Der Druckschalter war anfänglich am vorderen Rand des Tränkschälchens angebracht und wurde dort von den Tieren bei der Suche nach dem letzten Milchrest nach vorn gedrückt. Wenn alle Lämmer die Schalterbedienung beherrschten, wurde der Schalter vom Tränkschälchen etwa $10 \mathrm{~cm}$ seitlich entfernt angebracht. Dadurch wurden die Lämmer auf die Benutzung von Schaltern an der Lernapparatur vorbereitet. Die Schalter am Feldermonitor hatten einen horizontalen Abstand von $21 \mathrm{~cm}$ und einen vertikalen Abstand von $13 \mathrm{~cm}$ bei einer Gesamtbildfläche von $32 \times 25 \mathrm{~cm}$. Sie waren symmetrisch am äußeren Rand der Felder angeordnet.

Einige Tage vor der Umsetzung der Lämmer in die Box mit der Lernapparatur wurde die Tränkmilch durch Tränkwasser ersetzt, das ebenfalls nur nach Schalterbetätigung in $30 \mathrm{ml}$-Portionen ad libitum verabreicht wurde. 


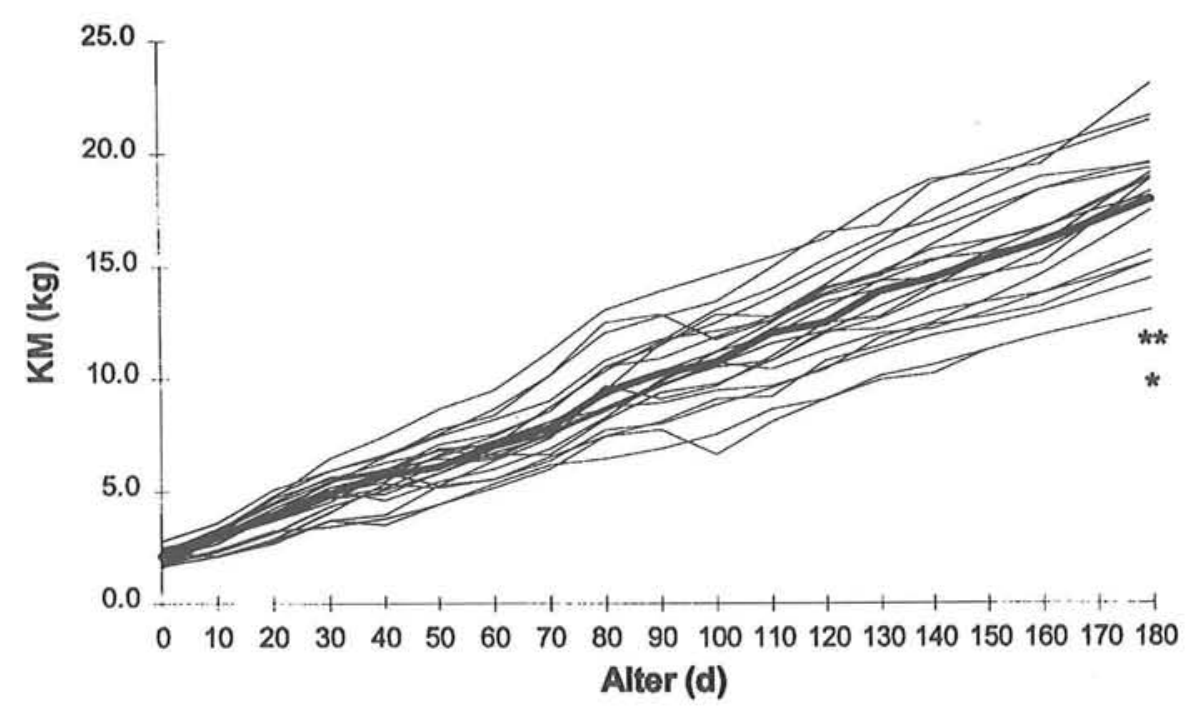

Abb. 3: Körpermasseentwicklung afrikanischer Zwergziegenlämmer (Development of body weight of dwarf goat lambs) (Einzeltiere der Versuchsgruppen 1 und 2 dünne Linien, Mittelwert starke Linie, "Mittelwert nach PETZOLD $=9,9 \mathrm{~kg}$, ${ }^{*}$ Mittelwert nach $\mathrm{JACOB}=11,8 \mathrm{~kg}$ ) (Thin lines: individuals of the groups 1 and 2 , bold line: average weights, "average from

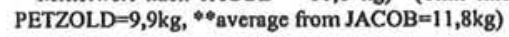

Der zeitliche Ablauf und die Dauer der drei Lerntests ist für die Versuchsgruppen in Tabelle 3 dargestellt.

Tabelle 3

Zeitplan zur Prüfung der visuellen Differenzierungsfähigkeit von Zwergziegen (Schedule for testing of visual differentiation ability of dwarf goats)

\begin{tabular}{cccccc}
\hline Test - Nr. & \multicolumn{2}{c}{ Versuchsgruppe 1 } & \multicolumn{2}{c}{ Versuchsgruppe 2 } \\
& Zeitraum & Dauer in Tagen & Zeitraum & Dauer in Tagen \\
\hline 1 & $25.02 .98-$ & 12 & $02.05 .98-$ & 13 \\
& 09.03 .98 & & 14.05 .98 & \\
& $10.03 .98-$ & 9 & $15.05 .98-$ & 20 \\
& 18.03 .98 & & 03.06 .98 & \\
& - & - & $04.06 .-16.06 .98$ & 13 \\
\hline
\end{tabular}

Die aufeinander folgenden Tests wechselten zu den in der Tabelle 3 aufgeführten Terminen automatisch um Mitternacht. Zu diesem Zeitpunkt wurden auch die Dateien der im Tagesverlauf angefallenen Datensätze abgeschlossen. Je Schalterbetätigung wurde ein Datensatz erzeugt, aus dem Datum, Zeitpunkt, Tiernummer, Feldnummer und Feldstatus (Steuerungsfunktion bzw. Bildpause) ersichtlich waren.

Die Aufbereitung der Daten erfolgte mit dem Programm MICROSOFT EXCEL 7.0.

Als Maß für den Lernerfolg eines Tieres an einem Testtag (LT\%) wurde der prozentuale Anteil richtiger (belohnter) Schalterbetätigungen an der Gesamtzahl Schalterbetätigungen gewählt. Als Maß für den Lernerfolg eines Tieres in einem längeren Zeitraum wurde die mittlere Rate der erfolgreichen Wahlversuche in den ersten 10 Tagen (LT10) gewählt. 
Im Testverlauf wurde für jedes Tier und jeden Tag geprüft, ob es alle verfügbaren Felder mit annähernd gleicher Häufigkeit gewählt hat. Dafür wurde auf Feldstetigkeit (FS), d.h. die unproportionale Häufigkeit der Wahl eines bestimmten Feldes, geprüft. Als Maß für die Feldstetigkeit wurde der Quotient aus der Gesamtzahl Schalterbetätigungen eines Feldes zum Erwartungswert des jeweiligen Feldes gebildet. Eine Feldstetigkeit wurde angenommen, wenn dieser Wert bei Wahlaufgaben aus zwei Feldern $\geq 1,5$ und bei Wahlaufgaben aus vier Feldern $\geq 2,0$ betrug.

\section{Ergebnisse}

Die Anzahl von Schalterbenutzungen (Aktionen) im Tagesverlauf zeigte erwartungsgemäß zwei herausragende Gipfel. Vormittags besuchten die Lämmer den Einzelstand gehäuft zwischen $7^{\circ \circ}$ und $10^{\circ \circ}$, nachmittags zwischen $14^{\circ \circ}$ und $17^{\circ \circ} \mathrm{Uhr}$ (Abb. 4). Während der späten Abend- und Nachtstunden wurde der Stand zwar in geringem Umfang, aber dennoch regelmäßig aufgesucht. Durch die ständig gegebene Möglichkeit des Besuches des Einzelstandes kam es bei der gewählten Gruppengröße nicht zu gegenseitigen Behinderungen. Der Tierwechsel verlief auch in den Zeiten der höchsten Besuchsfrequenzen ruhig und problemlos. Der Vergleich der Standbenutzung durch unterschiedlich schwere Lämmer ergab, daß die leichteren Lämmer einen annähernd gleichen Tagesrhythmus einhielten. Als Beispiel dafür ist in Abbildung 4 die Verteilung für drei Körpermasseklassen der Versuchsgruppe 2 im Test 1, Tag 1 - 10, dargestellt.



Abb. 4: Verteilung der Schalterbenutzungen im Tagesverlauf für unterschiedlich schwere Lämmer (Test 1, Tag 1 10, Versuchsgruppe 2) (Distribution of number of choices in the 24h-period for lambs of different body weight (Test 1 , day $1-10$, group 2))

Der erreichte Lernerfolg, gemessen am Anteil richtiger (belohnter) Schalterbetätigungen an der Gesamtzahl Schalterbetätigungen eines Tages (LT\%), ist in Abbildung 5 für Versuchsgruppe 2 dargestellt

Aus dem Vergleich der drei mittleren Lernkurven (starke Linien) wird deutlich, daß 


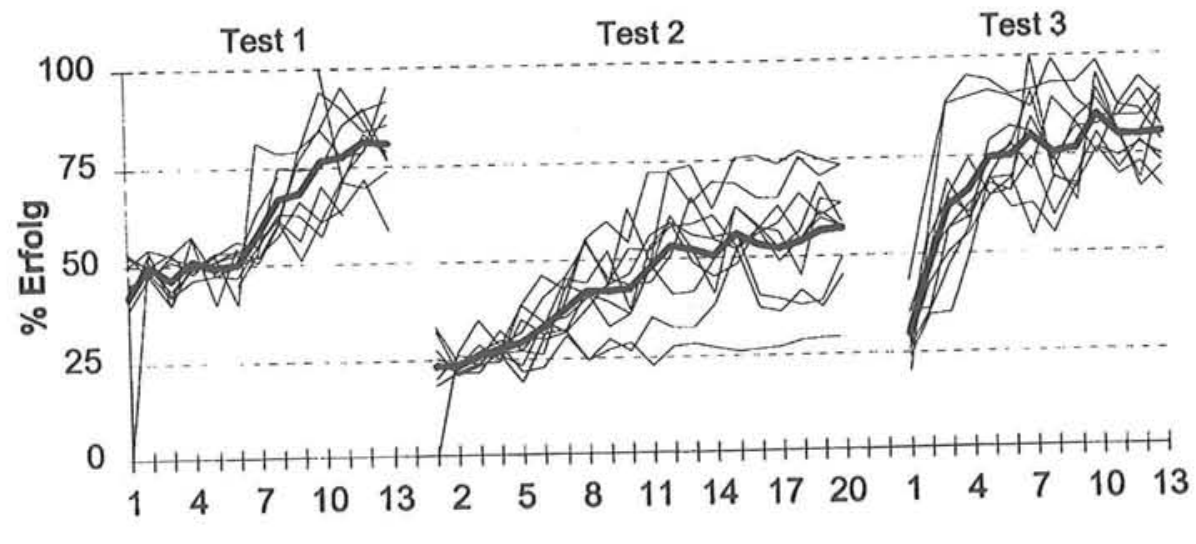

\section{Versuchstag}

Abb. 5: Individuelle und mittlere Lernkurven von Zwergziegen in 3 Differenzierungsaufgaben (Test 1, 2 und 3 der Versuchsgruppe 2, dünne Linien = Einzeltiere, starke Linien = Gruppenmittel) (Individual and mean learning curves of dwarf goats in 3 differentiation tests (Test 1,2 and 3 of group 2, thine lines-individuals, bold line=group average))

der Anstieg von Test zu Test früher einsetzt und steiler wird. Aus der Abbildung 5 ist weiter ersichtlich, daß alle Tiere (dünne Linien) einen Lernerfolg erreichten und daß zwischen den Tieren diesbezüglich beträchtliche Unterschiede bestanden. Die Variationskoeffizienten des Lernerfolges der ersten 10 Testtage (LT10) betrugen 14,6, 13,7 bzw. 13,5\% für die drei Tests.

Die Lernkurven der Tiere der Versuchsgruppe 1 sind in Abbildung 6 dargestellt.

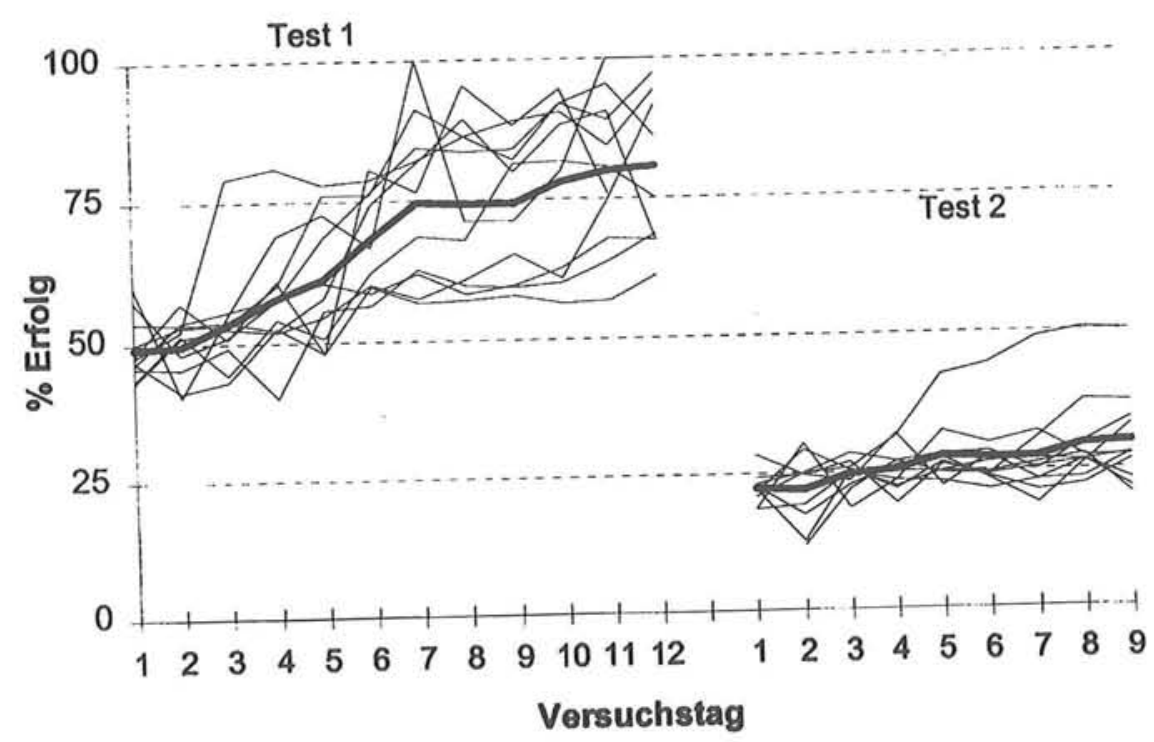

Abb. 6: Individuelle und mittlere Lernkurven von Zwergziegen in 2 Differenzienungsaufgaben (Test 1 und 2 der Versuchsgruppe 1, döne Linien = Einzeltiere, starke Linien = Gruppenmittel) (Individual and mean learning curves of dwarf goats in 2 differentiation tests (Test 1 and 2 of group 1, thine lines-individuals, bold line=group average)) 
Im Test 1 wurden von den Tieren dieser Versuchsgruppe ähnliche Ergebnisse erzielt, wie von denen der Versuchsgruppe 2. Im Test 2 dagegen wurde im Gruppenmittel bis zum 9. Tag kein signifikanter Lernerfolg erreicht. Mit einer Ausnahme hatten die Tiere für die Benutzung der Apparatur in diesem Zeitraum eine andere „Strategie“ gewählt.

Sie präferierten bestimmte Felder und erhielten durch den ständigen Wechsel der Position des belohnten Feldes hin und wieder eine Wasserportion.

Ein Vergleich der Häufigkeit von Feldstetigkeiten für die beiden Versuchsgruppen zeigt das Tabelle 4.

Tabelle 4

Häufigkeit von Feldstetigkeiten am ersten und letzten Testtag der Versuchsgruppen 1 und 2 (Frequency of 'field steadyness' at the first and last test day in the groups 1 and 2)

\begin{tabular}{|c|c|c|c|c|c|c|}
\hline \multirow[t]{2}{*}{ Feld Nr. } & \multicolumn{2}{|c|}{ Test 1} & \multicolumn{2}{|c|}{ Test 2} & \multicolumn{2}{|c|}{ Test 3} \\
\hline & 1. Tag & letzter Tag & 1. Tag & letzter Tag & 1. Tag & letzter Tag \\
\hline & \multicolumn{6}{|c|}{ Vers.-Gruppe $1(n=11)$} \\
\hline 1 & 2 & 3 & 0 & 0 & 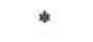 & \\
\hline 2 & 1 & 0 & 0 & 1 & & \\
\hline 3 & - & - & 3 & 2 & & \\
\hline 4 & - & - & 0 & 5 & & \\
\hline \multirow[t]{2}{*}{ gesamt } & 3 & 3 & 3 & 8 & & \\
\hline & \multicolumn{6}{|c|}{ Vers.-Gruppe 2. $(n=10)$} \\
\hline 1 & 0 & 0 & 0 & 0 & 0 & 0 \\
\hline 2 & 1 & 2 & 0 & 0 & 0 & 0 \\
\hline 3 & - & - & 0 & 2 & 3 & 0 \\
\hline 4 & - & - & 6 & 0 & 0 & 0 \\
\hline gesamt & 1 & 2 & 6 & 2 & 3 & 0 \\
\hline
\end{tabular}

Während bei der Versuchsgruppe 2 die Häufigkeit von Feldstetigkeiten im Test 2 von anfänglich 6 auf 2 zurückging (und im Test 3 von 3 auf 0), nahm die Häufigkeit bei der Versuchsgruppe 1 stark zu. Anfänglich bevorzugten im Test 2 drei Tiere das Feld 3. Der Quotient FS betrug 2,9. Diese Tiere wählten also Feld 3 fast dreimal mehr, als den Durchschnitt aller Felder. Am letzten Testtag bevorzugten 8 Tiere die Felder 2, 3 oder $4(\mathrm{FS}=2,9)$ und ein Tier die Felder drei und vier $(\mathrm{FS}=3,2)$ deutlich. Nur zwei Tiere lagen mit Feldstetigkeiten von 1,3 bzw. 1,5 unter dem Schwellwert. Die Tiere erhielten ihre Wasserportionen bei beständiger Wahl der bevorzugten Felder, weil durch den zufälligen Wechsel der Position des belohnten Schalters auch das von ihnen präferierte Feld hin und wieder den S+ Stimulus zeigte und dann auch die Wasserportion ermöglichte. Die Tiere erreichten ihre Belohnung nicht durch Beachtung des visuellen Stimulus, sondern durch häufigere Wahl der bevorzugten Felder. Erst nach diesem Ergebnis wurde die Software für zukünftige Tests so geändert und inzwischen erfolgreich erprobt, daß die stetige Wahl bestimmter Felder nicht mehr zur Belohnung führt.

\section{Diskussion}

Mit den Arbeiten von FEHRINGER (1979) und BALDWIN (1979) zum Lernverhalten von Ziegen bei visuellen Differenzierungsaufgaben und von BUCHENAUER und FRITSCH (1980) zum Farbsehvermögen von Ziegen war der Nachweis gelungen, daß 
Ziegen sowohl Farben unterscheiden wie auch andere visuelle Differenzierungsaufgaben lösen können. FEHRINGER (1979) kam nach dem Vergleich mit Ergebnissen anderer Autoren $\mathrm{zu}$ dem Schluß, daß Ziegen geringere Leistungen auf dem Gebiet des visuellen Lernens erbringen als beispielsweise Känguruhs, Zebras, Pferde, Esel, Elefanten oder Schweine. In ihren Experimenten erreichten die Ziegen im ersten Test erst nach 18 Tagen und 393 Versuchen die Kannphase. Das gewählte Zeichenpaar war ein schwarzes Plus-Zeichen für den belohnten Stimulus und ein schwarzer Kreis für den nicht belohnten Stimulus. Die gleiche Form hatten die Zeichen im ersten Test von BALDWIN (1979), jedoch waren bei ihm die Zeichen weiß auf schwarzem Hintergrund. In Anlehnung an diese Autoren wurde für den Test 1 das in Tabelle 1 beschriebene Zeichenpaar, ein Plus-Zeichen und ein Kreis, gewählt.

Die erzielten Ergebnisse bestätigen, daß Zwergziegen in der Lage sind, verschiedene visuelle Differenzierungsaufgaben zu lösen.

Im Unterschied zu den Beobachtungen von FEHRINGER (1979), wonach viele Tiere in der Wahlsituation gesträubtes Rückenhaar hatten (ein Ausdruck starker Erregung), zeigten die Ziegen im und am Einzelstand des Feldermonitors sehr selten Anzeichen von Erregung. Die Anzahl und die Verteilung der Schalterbenutzungen im Tagesverlauf wie auch im Verlauf aller Teste belegt, daß alle Tiere die Wahlanlage beliebig oft besuchen konnten. Es gab keine Anzeichen einer gegenseitigen Behinderung. Als Beleg dafür kann gelten, daß alle Tiere eine kontinuierlich gute Körpermasseentwicklung erreichten. Alle Tiere hatten eine im Vergleich zu Ergebnissen anderer Autoren (PETZOLD, 1980; JACOB, 1994) überdurchschnittlich gute Körpermasseentwicklung, die natürlich eine bedarfsgerechte Wasserversorgung voraussetzt.

Der Vergleich der mittleren Lernkurven der Versuchsgruppe 2 zeigt, daß bei den aufeinander folgenden Lerntests der Anstieg von Test zu Test früher einsetzt und steiler wird. Zwischen den Tieren bestanden beträchtliche Unterschiede im Lernerfolg. Die Variationskoeffizienten für das gewählte Maß des Lernerfolges (LT10) lagen mit 10 $20 \%$ im gleichen Bereich, wie er für viele andere quantitative Leistungsmerkmale bekannt ist.

Auf der Basis der erzielten Ergebnisse kann eingeschätzt werden, daß die entwickelte Lernapparatur Feldermonitor zur Prüfung des Lernverhaltens von in Gruppen gehaltenen Tieren geeignet ist.

Mit der Software, die in den vorgestellten Tests eingesetzt worden war, insbesondere mit dem Wechsel der Position des S+ Stimulus nach eingeschränkten Pseudozufallsfolgen, konnte jedoch nicht verhindert werden, daß auch eine andere "Strategie“ zur Erreichung von Belohnung entwickelt wurde. Die Tiere der Versuchsgruppe 1 folgten im Test 2 nicht dem visuellen Stimulus, sondern präferierten bestimmte Felder und wurden bei erhöhter Anzahl Schalterbetätigungen an diesen Feldern auch ausreichend mit Wasser versorgt. Zum Nachweis dieser Präferenzen erwies sich die Analyse der Feldstetigkeit (FS) als geeignet. Um in zukünftigen Lernexperimenten mit dem Feldermonitor die Entwicklung und Nutzung einer solchen „Strategie“ zu verhindern, wurde die Software entsprechend weiter entwickelt.

Mit den Ergebnissen und insbesondere mit der vorgestellten Methode sollen Anregun- 
gen für weitergehende Untersuchungen der sensorischen und kognitiven Leistungen von Nutztieren gegeben werden. Dadurch könnten Grundlagen für künftige Neuentwicklungen von Haltungs- und Fütterungssystemen entstehen.

Die Haltung von landwirtschaftlichen Nutztieren wird in zunehmendem Maße durch den Einsatz von Automaten für die Milch- und Futterversorgung, die Pflege und auch die Milchgewinnung verändert.

So werden Kälber schon überwiegend mit Tränkeautomaten versorgt, die Kraftfutterautomaten haben sich bei der Verbesserung der Fütterung von Rindern, Schweinen und Hühnern durchgesetzt und in europäischen Milchviehbetrieben werden gegenwärtig bereits über 300 automatische Melksysteme (Melkroboter) eingesetzt.

Für eine zweckmäßige Nutzung möglicher Interaktionen zwischen den Tieren und den entsprechenden Automaten fehlen aber wahrscheinlich die notwendigen wissenschaftlichen Grundlagen. Keines der bisher auf dem Markt angebotenen Gerätesysteme bezieht die als möglich erachtete Tier-Technik-Interaktion in die jeweiligen Prozesse ein. Eine Recherche der Publikationen zum Lernverhalten von Rindern (FRANZ, 1999) ergab, daß diesbezüglich relevante experimentelle Prüfungen von Rindern bisher nicht publiziert sind.

Potentielle Möglichkeiten für die Integration der Lernfähigkeit von Tieren werden beispielsweise hinsichtlich einer für die Automatenauslastung wünschenswerten Desynchronisation der Nutzungszeiträume durch die Tiere gesehen. Die Tiere müßten erlernen, entsprechend zeitlich versetzt gebotenen Signalen, die Automaten aufzusuchen.

Durch Automaten, die auf das individuelle Lernverhalten von Tieren reagieren, wäre eine Verbesserung der Tierumwelt im Sinne einer Beschäftigungstherapie (environmental enrichment) denkbar (WASHBURN et al., 1994; NICOL, 1996; KENDRICK, 1998).

Ein weiterer Aspekt für die Beurteilung von sensorischen und kognitiven Fähigkeiten von Nutztieren ergibt sich aus der heute gegebenen sehr hohen Vermehrungsrate bestimmter Zuchttiere durch die künstliche Besamung oder andere Biotechniken. Für solche Tiere sollte durch geeignete Prüfungen gesichert werden, daß bisher unerkannte sensorische Defekte nicht übermäßig verbreitet werden. Es ist gegenwärtig nicht bekannt, welche Variabilität für sensorische und kognitive Leistungen von Nutztieren besteht.

Auch das Generalisationsvermögen von Nutztieren erscheint für zukünftige Haltungsumwelten von großer Bedeutung. Beispielsweise könnten beim Einsatz von Automaten verschiedener Hersteller in unterschiedlichen Aufzuchtabschnitten oder Betrieben die Tiere mit verschiedenen Stimuli konfrontiert werden. Eine Einschätzung der Auswirkungen auf die Tiere oder ihrer Anpassungsmöglichkeiten fehlt bisher.

\section{Literatur}

ANDREWS, J.S.; JANSEN, J.H.M.:

Matching to sample in rats using projected visual stimuli. Neuroscience Research Communications, 18 (1996) 2, 115-123

BALDWIN, B.A.:

Operant studies on shape discrimination in goats. Physiol. Behav., 23 (1979), 455-459 
BUCHENAUER, D.; FRITSCH, B.:

Zum Farbsehvermögen von Hausziegen (Capra hircus L.). Z. Tierpsychol., 53 (1980), 225-230

COOK, R.G.; KIMBERLEY, K.C.; CAVOTO, R.:

Same-Different texture discrimination and concept learning by pigeons. J. Exp. Psychology. Animal Behavior Proc. 21 (1995) 3, 253-260

DASSER, $\mathrm{V}$.:

Slides of group members as representations of the real animals (Macaca fascicularis). Ethology 76 (1987), 65-73

EMMERTON, J.:

Pigeons discrimination of colour proportions in video displays. Proceedings of the 26rd Göttingen Neurobiology Conference, Georg Thieme Verlag Stuttgart-New York (1998), II, 528

ENNACEUR, A.; AGGLETON, J.P.; FRAY, P.J.:

Delayed non matching to sample in a novel automated visuel memory apparatus using mixed retention intervals. Neuroscience Research Communications, 20 (1997) 2, 103-111

FEHRINGER, $\mathrm{CH}$.:

Versuche zum visuellen Lernen bei Afrikanischen Zweigziegen (Capra hircus L.). Justus-Liebig-Universität Gießen, Diss., 1979

FEULNER, A.:

Der Einfluß von Transferlernen auf die Stabilităt der Verknüpfung sensorischer und motorischer Gedächtnisinhalte. Philipps-Universität Marburg, Diss., 1993

FRANZ, H.:

Methode zur Untersuchung der Lernfahigkeit von Kallbern in Gruppenhaltung und Ergebnisse bei visuellen Differenzierungsaufgaben. Arch. Tierz., Dummerstorf 42 (1999) 3, 241-254

HOPKINS, W.D.; WASHBURN, D.A.; HYATT, C.W.:

Video-task acquisition in rhesus monkeys (Macaca mulatta) and chimpanzees (Pan troglodytes): A comparative analysis. Div. Behav. Biol. Yerkes Regional Primate Res. Cent., Emory, Univ., Atlanta, Primates 37 (1996), 197-206

JACOB, U.: Ziegenhaltung bei Kleinbauern in Burundi. Humboldt-Universităt Berlin, Diss., 1995

KENDRICK. K.M.: Intelligent perception. Applied Animal Behaviour Science, 57 (1998), 213-231

MEIER, M.; REINERMANN, R.; WARLICH, J.; MANTEUFFEL, G.:

An automated training device for pattern discrimination learning of group-housed gerbils. Physiology \& Behavior, 63 (1997) 4, 397-498

NICOL, C.J.: Farm animal cognition. Animal Science, 62 (1996), 375-391

PETZOLD, G.:

Metrische Untersuchungen zur Jugendentwicklung afrikanischer Zwergziegen in Zoologischen Gärten. Milu, Berlin 5 (1980); 5-20

WASHBURN, D.A.; HARPER, S.; RUMBAUGH, D.M.:

Computer-task testing of rhesus monkeys (Macaca mulatta) in the social milieu. Sonny Carter Life Sci. Lab., Psychol., Georgia State Univ., Plaza Atlanta, Primates 35 (1994), 343-351

WASHBURN, D.A.; RUMBAUGH, D.M.:

Impaired performance from brief social isolation of rhesus monkeys (Macaca mulatta): A multiple video-task assessment. J. Comparative Psychology 105 (1991), 145-151

Eingegangen: 14.09 .1998

Akzeptiert: 23.04 .1999

Anschriften der Verfasser

Dr. HARTMUT FRANZ

Forschungsinstitut für die Biologie landwirtschaftlicher Nutztiere (FBN)

Wilhelm-Stahl-Allee 2

D-18196 Dummerstorf

Dr.-Ing. HORST REICHART

Forschungszentrum für Biosystemtechnik und Biomaterialien

Friedrich-Barnewitz-Straße 4

D-18119 Rostock-Warnemünde

E-Mail: franz@fbn-dummerstorf.de 


\title{
Buchbesprechung
}

\author{
Krankheiten des Pferdes - Ein Leitfaden für Studium und Praxis
}

\author{
HANS-JÜRGEN WINTZER (Hrsg.) \\ Mit Beiträgen von W. BISPING, H.-H. FREY, H. GERBER, P. GLATZEL, H. KELLER, A. KRÄHENMANN, \\ W. KRAFT, J. KRONEMANN, H. LUDWIG, H.-J. WINTZER
}

2. vollständig überarbeitete Auflage, 608 Seiten, 457 Abbildungen davon 194 auf 24 Farbtafeln, 21Tabellen, Parey Buchverlag, Berlin, im Blackwell Wissenschaftsverlag Berlin, Wien, 1997, ISBN 3-8263-3031-5, $198,00 \mathrm{DM}, 1445,00 \mathrm{OS}, 182,50 \mathrm{SFr}$

Dieses Standardwerk über die Krankheiten des Pferdes liegt in 2. überarbeiteter Auflage vor. Der Herausgeber, unterstützt von kompetenten Fachvertretern aus Deutschland, den Niederlanden und der Schweiz, hat dieses Standardwerk für Studium und Praxis sowohl im Umfang als auch in der Abbildungsanzahl erweitert und unter Einbeziehung neuester Erkenntnisse in der Diagnostik und Therapie vollständig überarbeitet vorgelegt. Eine besonders breite Überarbeitung erfuhren die Abschnitte über die Krankheiten des Atmungsapparates, der Bauchhöhlenorgane und des Zentralnervensystems. Mit der Neuauflage dieses Buches wird einem allgemeinen Trend, nämlich der stärkeren Zuwendung in der tierärztlichen Praxis zum Pferd, entsprochen. Es ist kein Zufall, daß neben einer umfangreich vorliegenden Fachliteratur in der Tiermedizin zur Chirurgie beim Pferd, in diesem Standardwerk vor allem den inneren Krankheiten des Pferdes und den Fertilitätsproblemen breiterer Raum gewidmet wird, was dieses Buch besonders auszeichnet.

Am Aufbau und der Einteilung des Buches wurden keine Änderungen vorgenommen. Die dreizehn Hauptabschnitte enthalten die Besprechung der Krankheiten des Atmungsapparates, des kardiovaskulären Systems, des Verdauungsapparates, der Harnorgane, der Geschlechtsorgane, der Gliedmaßen, der Weichteile, des Kopfes, des Rumpfes, der Haut, des Zentralnervensystems, die Infektionskrankheiten, Stoffwechselkrankheiten und Vergiftungen.

Die einzelnen Hauptabschnitte sind sehr breit gefächert gegliedert und können dadurch sehr speziell informieren. Die Beschreibungen der einzelnen Krankheiten sind didaktisch gut aufbereitet. Dargestellt werden u.a. die einzelnen Krankheiten, allgemein diagnostische Gesichtspunkte und es sind umfangreiche Informationen u.a. zu den Diagnosemethoden, zur Therapie und Prognose enthalten. Das hervorragende z.T. farbige Bildmaterial sowie zahlreiche Abbildungen und Tabellen untersetzen die auf dem neuesten Stand beruhenden, verständlichen und praxisrelevanten Textaussagen. Erwăhnt seien die jedem Unterabschnitt angefitgten umfangreichen Literaturangaben. Ein abschließendes Sachverzeichnis erleichtert die Arbeit mit diesem Buch.

Dieser vorliegende Leitfaden für Studierende und Praktiker entspricht einem sehr hohen Standard veterinärmedizinischer Fachliteratur und bedarf, bezogen auf seinen fachspezifischen Inhalt, die Autoren und den Verlag, eigentlich keiner besonderen Empfehlung. 